\title{
Increasing the yield of the high-resolution anoscopy with endoscopic assistance: A gastroenterologist's perspective
}

\author{
Harish Patel*, Hassan Tariq, Jasbir Makker, Rafeeq Ahmed, Mariela Glandt, Peter Bhandari, Vamshidhar Vootla, Chaitanya Chandrala and \\ Sridhar Chilimuri
}

Division of Gastroenterology, Department of Medicine BronxCare Health System, Bronx, New York 10457, USA

\begin{abstract}
Background: There is an increase in the rates of the Human Papilloma virus (HPV) related anal cancer in human immunodeficiency virus (HIV) positive individuals. The anal dysplasia screening is offered to the individuals at risk and anal cytology is one of the screening modalities. High resolution anoscopy (HRA) is performed for the further evaluation of the abnormal anal cytology. HRA plays a key role in the identification and management of high-grade squamous intraepithelial lesion (HSIL). Yield of HRA to detect HSIL in patients with HSIL on anal cytology can be affected with factors like difficult anal anatomy and use of microscope respectively. Acquiring HRA skills and using a microscope to increase the yield has a steep learning curve, and hence leaving most gastroenterologists untrained in this examination.
\end{abstract}

Objectives: In this retrospective review, we intend to describe endoscopy assisted high-resolution anoscopy (eHRA) and its yield in detecting HSIL in a small group of patients.

Methods: We performed a retrospective review of all HRA performed between the study period July 2015 to June 2018 . The indications for all the HRA were reviewed. The yield of the HRA for the patient with HSIL anal cytology was analyzed. Records of patients with negative initial HRA, who underwent Colonoscopy for other reasons and had a simultaneous eHRA performed, were reviewed. The utility of the eHRA in terms of its yield to detect HSIL was computed.

Results: We performed a total of 237 high-resolution anoscopy on 197 unique patients, from July 2015 to June 2018 . A total of 21 (14\%) patients who had HSIL underwent HRA. On HRA, 14 out of 21 (70\%) patients had HSIL. Among the remaining 7 patients who had no high-grade anal lesion on the index HRA; three patients had no aceto-whitness on HRA and 4 patients had a non-dysplastic pathology on biopsies. 6 patients among this group with negative index HRA underwent eHRA. 5 out of these 6 patients (83\%) were noted to have HSIL and 1 patient had LSIL. Hence, eHRA increased the initial HSIL yield of $70 \%$ (14 out of 21 ) to $95 \%$ (19 out of 21$)$.

Conclusions: The endoscopy assisted high resolution anoscopy can increase the yield of detecting HSIL. It overcomes the gastroenterologist's limitation due to lack of versatility and experience with the viewing microscope, as well as overcome patient limiting factors like difficult anal anatomy and incomplete examination due to patient discomfort.

\section{Introduction}

Colonoscopy is the best tool for the colorectal cancer screening. It is a chartered territory of gastroenterologists [1]. The sole concept is identification of lesions that are precursors to colon cancer. Highresolution anoscopy (HRA) as performed for the evaluation of anal dysplasia is an unchartered territory of the gastroenterologist [2]. HRA is mainly performed by physicians with expertise in infectious diseases, gynecology and colon \& rectal surgery. Patients with HIV, MSM (men having sex with men) and woman with high-grade genital lesions should be evaluated for human papillomavirus (HPV) related anal dysplasia [3]. Initial evaluation includes anal cytology and subsequent follow up of abnormal cytology with the HRA. Based on degree of dysplasia in the anal epithelium, lesions are classified either as low grade squamous intraepithelial lesion (LSIL) or high-grade squamous intraepithelial lesion (HSIL).

Patients with the HSIL on the initial screening anal cytology are more likely to have HSIL on HRA [4,5]. HIV positive patients with HSIL but subsequent HRA rendering no anal lesion, need follow-up in one year [4].

HRA has a steep learning curve. The procedure includes application of $5 \%$ acetic acid to the anal area which makes tissues with dysplasia turn white in color and thus differentiating normal from abnormal mucosa. Further examination of areas of uncertainty can be done after application of Lugol's iodine which turns cells harboring dysplasia yellow whereas normal cells turn brown [6]. Physicians well versed with the colposcope have a higher chance of identifying a lesion on HRA due to their experience with colposcopic examination of cervical disease. Anal and cervical examinations may seem similar, but the difficult anatomy of anal canal and the steep learning curve for HRA increases the likelihood of missing the dysplastic anal lesions. Gastroenterologists are well versed with use of the endoscope for luminal gastrointestinal evaluation. We utilized a novel technique of endoscopy assisted highresolution anoscopy (eHRA) for anal examination. We believe that

${ }^{\star}$ Correspondence to: Harish Patel, Division of Gastroenterology, Department of Medicine Bronx Care Health System, Bronx, New York, USA, E-Mail: hpatel@ bronxcare.org

Key words: endoscopy and high resolution anoscopy, gastroenterologist and high resolution anoscopy, anal cancer, high-grade squamous intraepithelial lesions, anal dysplasia screening

Received: June 18, 2019; Accepted: July 04, 2019; Published: July 08, 2019 
utilizing endoscopy assistance for HRA increases the yield as also supported by our data presented later. Yield possibly increases due to better light source and magnification provided by the endoscopy assistance. This technique also helps an untrained eye to uncover lesions which may not be possible otherwise. We present here our experience with this technique in a small group of patients. To our knowledge this protocol has never been described before and it is an innovation to the anal dysplasia evaluation.

\section{Methods}

A physician trained and certified in HRA by American Society for Colposcopy and Cervical Pathology (ASCCP), examined all the study patients and performed the anoscopy and colonoscopy. Initial screening of high-risk patients is routinely performed at our institute by patient's primary care and infectious disease physician. If indicated the anal cytology is performed. Patients with abnormal anal cytology and other high-risk features are referred to the gastroenterologist practice for HRA. Results reported here in the study are obtained from all the HRA performed during the study period. The initial evaluation was performed for the Quality and Performance Improvement project. Study was also approved by BronxCare Health System Institutional Review Board Committee.

\section{Study population}

All the patients who underwent HRA during a 3-year study period from July 2015 to June 2018 were included in the initial review. We studied indications for HRA and the pathology of the anal biopsy. The patients with HSIL on the screening anal cytology were identified. The outcomes of HRA in terms of ability to identify the high-grade anal lesion in these patients with HSIL on anal cytology were noted. HRA examination was considered to be negative if there was no high-grade anal lesion identified. In HIV positive patients with HSIL on cytology but subsequent HRA rendering no anal lesion needs follow-up in one year [4]. However, a six-month follow-up HRA was performed at our institution. Those patients who required colonoscopy for other reasons were offered simultaneous HRA in the endoscopy unit. From this group of patients with negative HRA finding and HSIL on anal cytology, patients who underwent colonoscopy and simultaneous eHRA were reviewed. The eHRA in these patients was performed prior to performing a complete colonoscopy examination. Six patients who had the eHRA are described later in this review.

\section{Study Definition: Yield of the high resolution anoscopy}

The yield of HRA pertains to its ability to identify the high-grade anal lesion in a patient with HSIL anal cytology. Statistically it is defined as the percentage of total number of patients with HSIL anal cytology who were diagnosed with the high-grade anal lesion on HRA. Prior studies have demonstrated a yield of around $90 \%$ with high resolution anoscopy in such patients [5]. However, in our patients we did not notice this high yield on our initial HRA examination, hence prompting us to repeat HRA sooner than recommended 1 year. We intend to study the yield of HRA performed with Colonoscopy assistance in these repeat examinations.

\section{Procedure description: Endoscopy assisted high resolution anoscopy (eHRA)}

The term eHRA is coined to describe the HRA performed under colonoscopy guidance. The steps for HRA examination essentially remained the same. The examination is performed in the left lateral position. The patient is prepped for the Colonoscopy with full bowel prep. The eHRA was performed prior to proceeding with complete colonoscopy examination. We used single beveled, disposable plastic anoscope. The surgical marker was used to mark one side of the anoscope (Figure 1). At the time of anoscope insertion, this marker is aligned with right side of anal canal and during the examination this marker provides topographic orientation of the anal canal lesions. The Olmypus X190 series high definition pediatric colonoscopes were utilized for the eHRA.

The visual inspection and digital rectal examination are performed as a first step to identify any palpable lesions and to assess the anal sphincter tone. The cotton applicator soaked in 3\% acetic acid (Figure 2 ) is applied to anal canal for 2-minute duration. The anoscope in then inserted with the marker opposing the right side (Figure 3). The pediatric colonoscope with the diameter of 11.8 millimeter $(\mathrm{mm})$ can be successfully inserted through the anoscope with no resistance (Figure 4). The colonoscope is torqued to orient the anoscope marker to right anal canal, this is depicted in the screen at 12 O'clock position. As the initial part of the examination the area opposing on the anoscope is examined for any identifiable aceto-whiteness. Then anoscope is directed towards the squamo-columnar junction to identify and biopsy the mucosa with the aceto-whiteness. After the initial anoscopy examination completion, the anoscope is retrieved and the colonoscope is inserted to perform the colonoscopy.

\section{Results}

We performed a total of 237 high-resolution anoscopy procedures on 197 unique patients from July 2015 to June 2018 for evaluation of abnormal anal cytology. A total of 21 of these patients (14\%) had HSIL and underwent HRA. 14 out of these 21 patients $(70 \%)$ had a positive finding of dysplastic anal pathology on the HRA (Chart 1). Remaining 7 patients who had HSIL anal cytology did not have any dysplastic lesion on the index HRA examination- 3 patients had no aceto-whiteness and 4 patients had aceto-whitness but no significant lesion on the pathology. 6 out of this group of 7 patients with HSIL anal cytology but negative initial HRA were scheduled for colonoscopy for evaluation of unrelated additional colorectal symptoms. The eHRA was discussed with them in view of the possibility of the missed anal dysplasia. Prior to the colonoscopy, eHRA was performed. There was a total of $5(83 \%)$ patients with HSIL and one with LSIL on this second HRA examination performed under endoscopy guidance. Hence, eHRA increased the initial HSIL yield of $70 \%$ (14 out of 21 ) to $95 \%$ (19 out of 21 ).

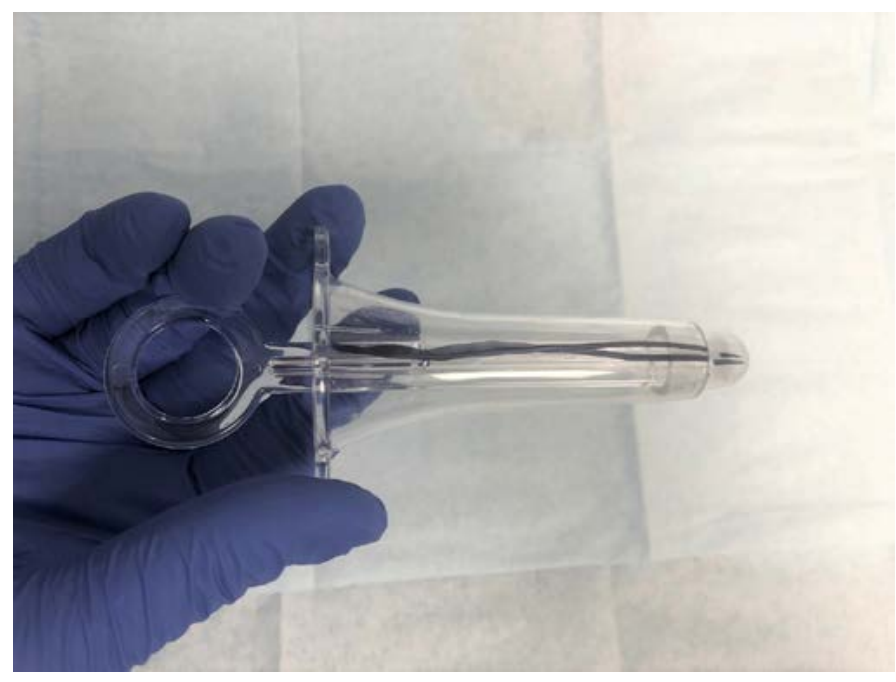

Figure 1. Disposable anoscope with marking 


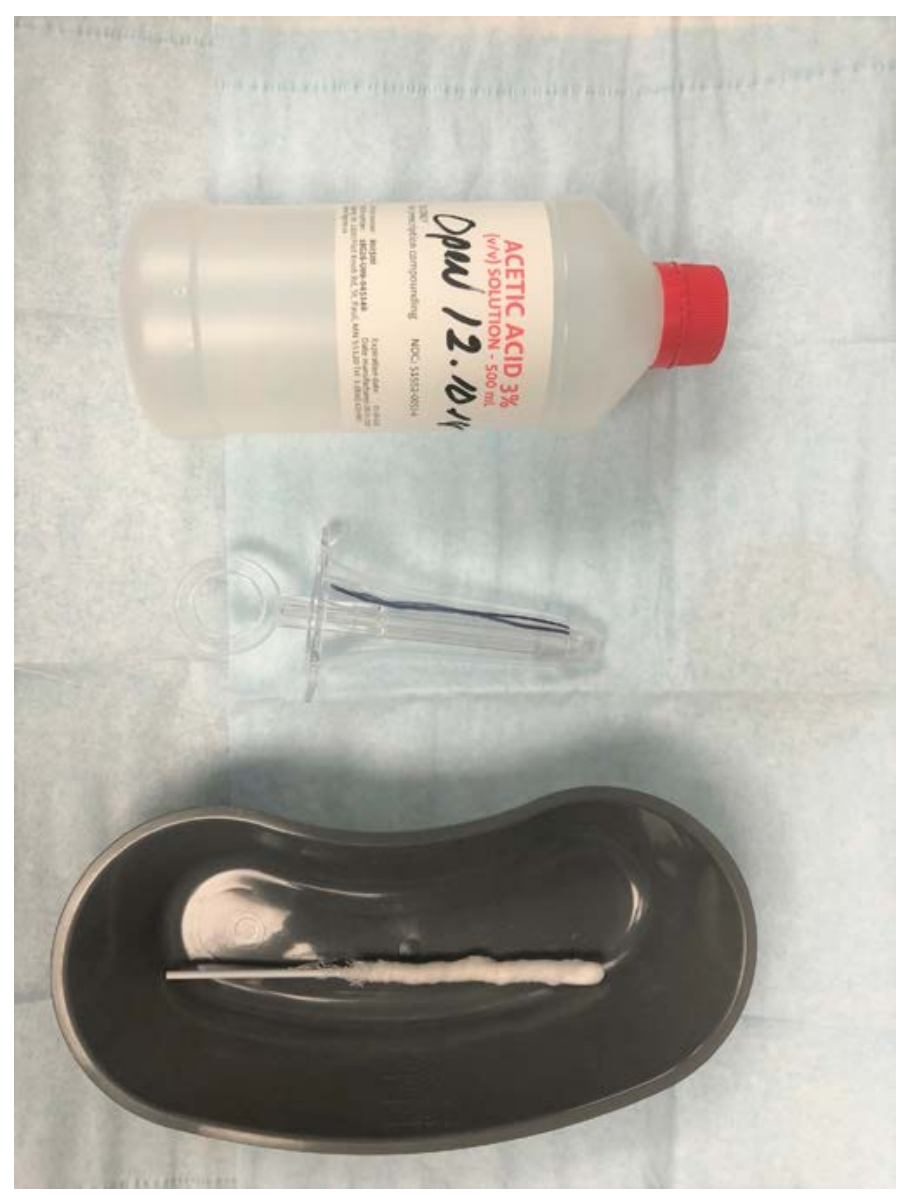

Figure 2. Cotton applicator and acetic acid (3\%)

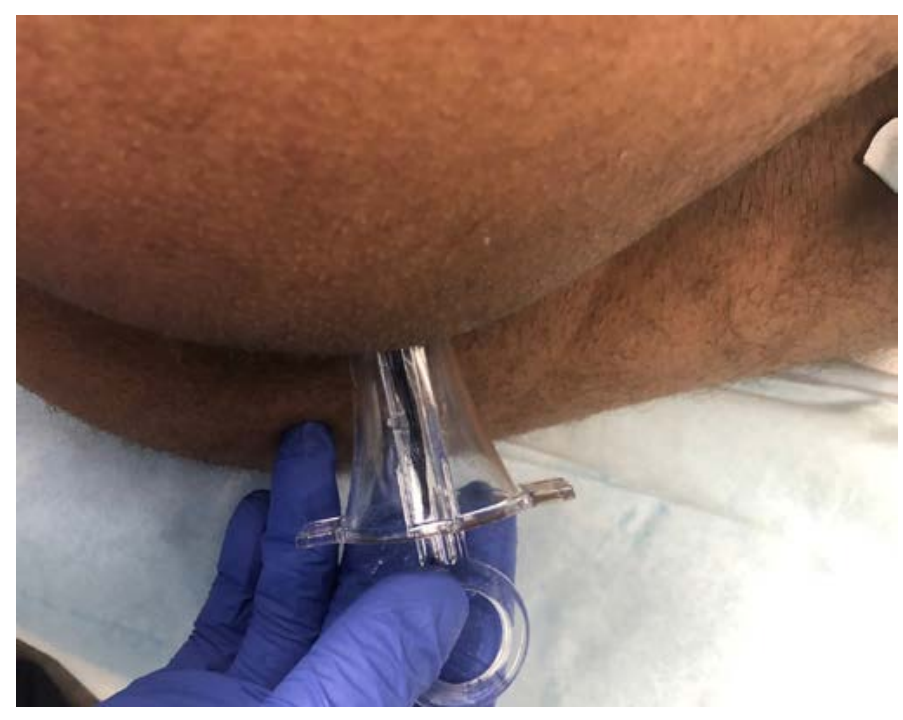

Figure 3. Demonstrates anscope insertion with marker opposed to right anal canal

\section{Case Descriptions}

\section{Case 1}

A 51 year-old-man with the co-morbid conditions of HIV and CD4 count of 400 cells $/ \mathrm{mm}^{3}$ had HSIL on the initial screening anal cytology. The initial anal examination revealed hemorrhoids. HRA was technically difficult due to presence of large hemorrhoids and no aceto-whiteness was perceived. The patient was then scheduled for the screening colonoscopy and eHRA which revealed right sided acetowhiteness, biopsy of which revealed HSIL. The patient was referred to Colo-rectal Surgery for the further management.

\section{Case 2}

A 31-year man HIV negative, MSM was referred for HSIL on screening anal cytology. The HRA revealed left distal aceto-whiteness, Lugol's stain positive anal lesion and the biopsy revealed a LSIL. The patient had significant discomfort on this initial HRA. The concomitant anal cytology again revealed HSIL. There were no findings of anal fissure. He underwent colonoscopy for evaluation of colitis seen on computerized tomography (CAT) scan of abdomen. The eHRA revealed posterior anal canal aceto-whiteness and the pathology was reported as HSIL. He was referred to Colo-rectal Surgery for the further evaluation.

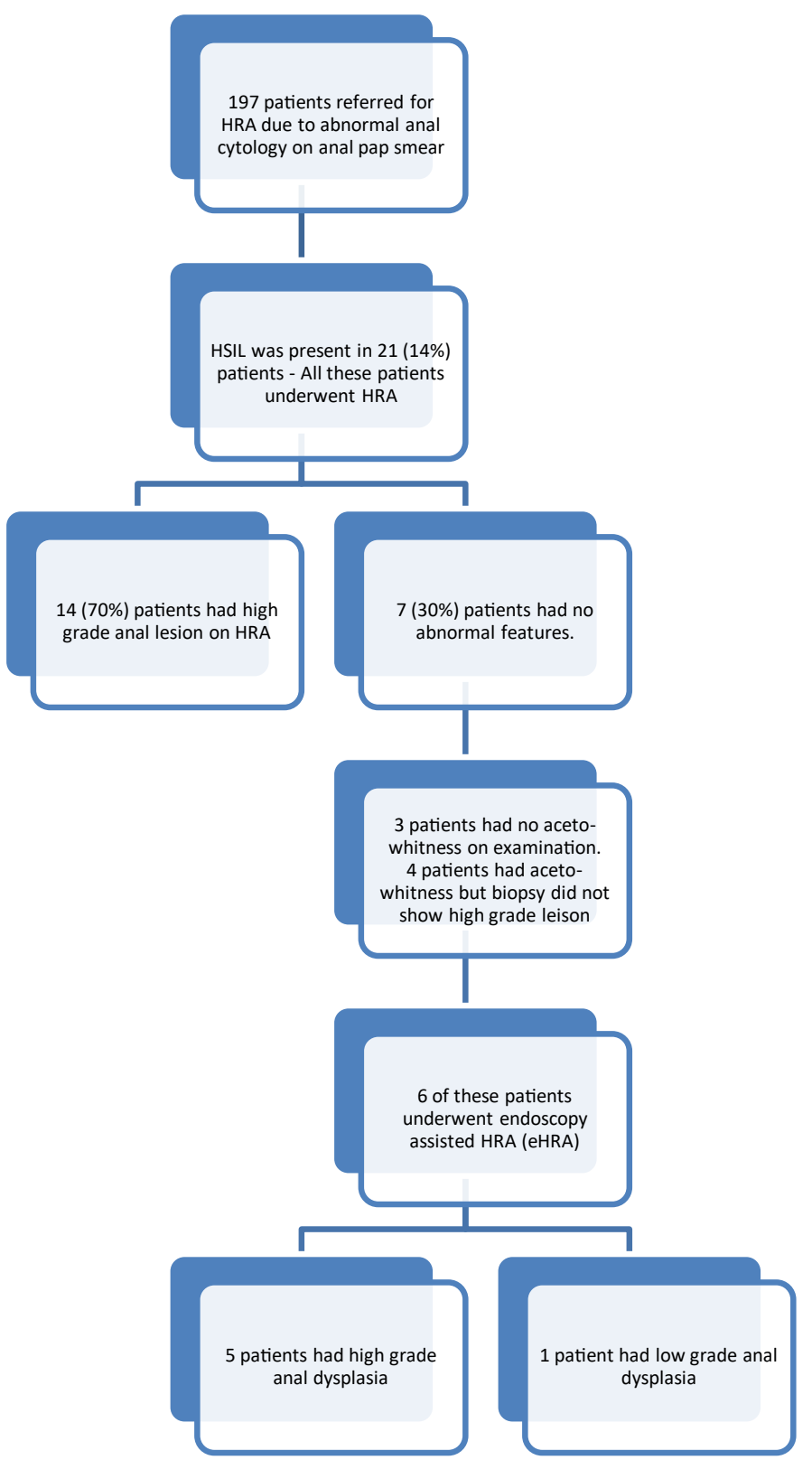

Chart 1. Demonstrates flow chart for patients with HSIL on anal cytology and yield of HRA and eHRA is diagnosis of high-grade anal lesion 


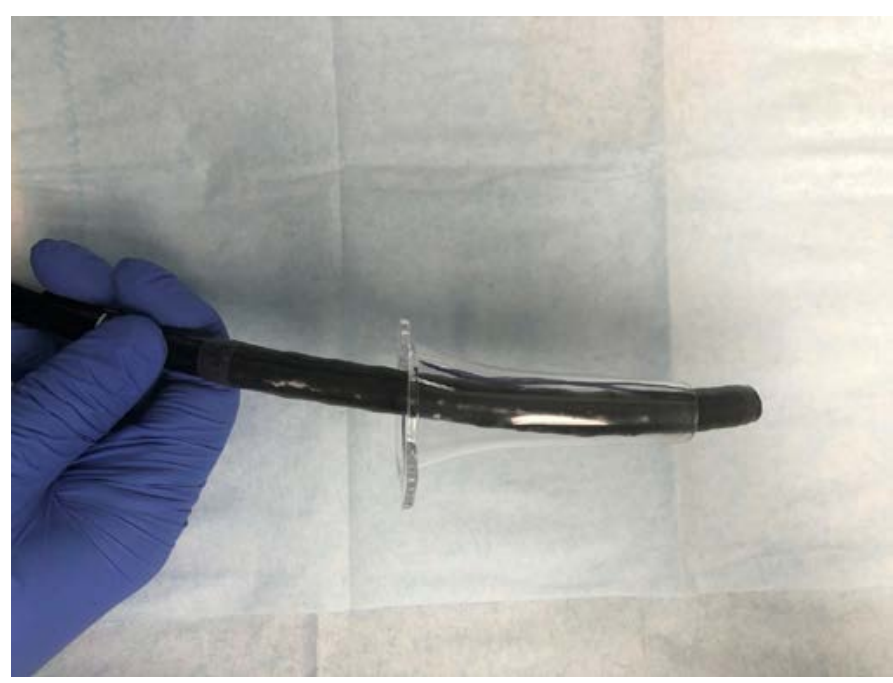

Figure 4. Demonstrates colonoscope insertion through the anoscope

\section{Case 3}

A 32-year-old woman whose co-morbid conditions included HIV and being a smoker, with no prior history of high-grade cervical lesions was referred due to findings of HSIL on the anal cytology. She had multiple sexual partners, denied receptive anal intercourse. HRA was technically difficult due to patient experiencing significant discomfort during the procedure. There was no aceto-whiteness noted. The patient did not agree to repeat the evaluation. She underwent colonoscopy for rectal bleeding; eHRA revealed left anterior aceto-whiteness and the biopsy revealed HSIL. She was referred to Gynecologic Oncology and underwent electrodessication of the left anterior lesion. Her colonoscopy revealed hemorrhoids, which was likely the etiology of rectal bleeding.

\section{Case 4}

A 61-year-old man with a history of prostate cancer noted to have anal warts underwent anal cytology test which revealed HSIL. The HRA revealed the hypertrophied anal papillae, in the right distal region of anal canal the pathology revealed LSIL. Sigmoidoscopy was performed for management of radiation proctopathy. eHRA revealed aceto-white lesion in the posterior aspect of anal canal and the biopsy revealed HSIL. The patient was referred to Colo-rectal Surgery for the further management.

\section{Case 5}

A 54-year man, HIV negative, non-smoker, was evaluated after his first screening colonoscopy demonstrating prominent hypertrophied anal papillae with biopsy revealing Anal Intraepithelial Neoplasia-I. This first colonoscopy was limited due to poor bowel preparation. The anal cytology revealed the HSIL and the biopsy of the aceto-whiteness in the anterior region on the subsequent HRA revealed atypical squamous cell epithelium. The eHRA was performed and it revealed right anterior HSIL. Subsequently, patient relocated to another state and did not return to our institution for the further management.

\section{Case 6}

A 44-year-old man with comorbid condition of HIV, smoker, heterosexual, noted to have HSIL on the anal cytology, later HRA did not show any aceto-whiteness and the concomitant anal cytology was reported as atypical squamous cells of undetermined significance
(ASCUS). A colonoscopy was planned for the evaluation of the colonic stenosis as seen on the CT abdomen. The eHRA revealed the posterior aceto-whiteness, with HSIL on the pathology. The patient was referred to Colo-rectal Surgery for the further management.

\section{Discussion}

Squamous cell carcinoma of the anal canal (SCCA) is the most common anal cancer [7]. Human Papilloma Virus (HPV) is known to be associated with the SCCA. With the change in sexual behavior, there has been an increase in the prevalence of HPV infection [8]. There is a dramatic increase in the incidence of SCCA, with more patients being diagnosed at a younger age [9]. The prolonged immunocompromised status resulting from the organ transplant and Human Immunodeficiency Virus (HIV) infection may have attributed to this increased incidence of SCCA [10]. Anal cancer is the most common non-AIDS defining cancer with 19 times higher risk in patients with HIV as compared with non-HIV individuals [11].

The squamo-columnar junction at the anal canal, histologically similar to cervix, is a high cell turnover zone. There are some immunophenotype distinctions in the HPV related carcinogenesis at these two sites [12]. The dysplastic alteration from the HPV infection are considered to be the precursors for the invasive squamous cell carcinoma [13]. The spectrum of the anal dysplasia ranges from LSIL to HSIL [14]. In patients with HIV, despite the use of the Highly Active Anti-Retroviral Therapy (HAART), fifty percent of the patients with HSIL progressed to anal cancer [15]. On the other hand, there has been a $50 \%$ reduction in the rates of newly diagnosed cervical cancer [16]. The most important factor contributing towards this difference at two sites is the availability and feasibility of widespread cervical cancer screening and further management of the cervical HSIL and high risk LSIL [17]. Currently, benefits of treating anal LSIL and HSIL is not clearly established and moreover not approved by all insurance carriers. Hence, Palefsky et al, have initiated "The ANCHOR (Anal Cancer HSIL Outcomes Research) Study" where they intend to show the benefits of treating such dysplastic anal lesions by comparing the outcomes in two groups with and without treatment. However, any such treatment to be possible and effective will require training of physicians in HRA to be able to identify a lesion.

The target screening population includes patients with HIV, MSM, woman with high-grade genital lesion or cancer, and organ transplant recipients [18]. There is uncertainty about anal dysplasia screening in patients with non-HIV related immunosuppression, like those on chemotherapy and receiving biological agents for inflammatory bowel disease. The digital anorectal exam (DARE), anal cytology evaluation, HPV testing and the HRA have been evaluated as the screening modality for anal dysplasia [19-22]. Although providers have adopted the screening protocol from the University of San Francisco, the optimal approach is yet to be defined [4]. Those with anogential warts, anal pain and abnormal DARE should proceed to HRA. In others, anal cytology is performed, and it is evaluated as per the Bethesda System classification [23]. The patients with abnormalities of the anal cytology are further evaluated with HRA.

Gastrointestinal complaints are common in patients with HIV. Gastroenterologists are involved in the management of gastrointestinal opportunistic infections, inflammatory and direct mucosal pathologies affecting the gut $[24,25]$. Majority of the gastrointestinal manifestations of HIV are related to the level of the immunosuppression, as evaluated by the CD4 count in the HIV positive individuals [26]. The optimal management of HIV with HAART has dramatically decreased the 
opportunistic and the luminal gastrointestinal disease incidence [27]. However, despite HAART the rates of the HPV related anal cancer have increased [15]. The early diagnosis and primary preventive strategies for anal cancer would have significant impact on the health of HIV positive population [28]. HRA remains the corner stone for diagnosis of high-grade anal lesions. The number of the providers trained in performing HRA is limited. It is considered the territory of the proctologist, gynecologist and the infectious disease physicians. The use of the HRA is not included in most gastroenterology-training curriculums. However, with proper training, a gastroenterologist well versed in the diagnostic and therapeutic interventions can acquire the HRA technique.

HRA has a steep learning curve. The procedure includes application of acetic acid (3\% or 5\%), and subsequent evaluation of the anal squamo-columnar junction and perianal area for the acetowhiteness. The Lugol's Iodine is applied for the better delineation of the anal lesions $[6,22]$. The anal examination is performed with the anoscope at 16X magnification and the area of the interest is examined at $25 \mathrm{X}$ magnification. The positive findings on the anal examination are biopsied. If the HRA is performed as a result of the HSIL anal cytology, then yield of $90 \%$ is expected. Although, International Anal Neoplasia Society (IANS) has set clear standards for practicing HRA, yield is not always high in all practices due to few shortcomings [29]. In our experience, HRA may have provider, instrument and patient related factors that would affect its yield.

The concept of using a binocular microscope in the HRA has been adopted from the prior experience form colposcopy. The focal length in most colposcopes is around 30 centimeters $(\mathrm{cm})$. It is challenging to have binocular microscope focus on the anal area while sustaining the pressure on the anoscope and maintaining the anal-anoscopemicroscope axis. The video colposcope, though has an autofocus at 25 to $30 \mathrm{~cm}$, has the limitation of view getting obscured during instrumentation. The video ProctoStation (Balmer Medical') can certainly overcome the limitation of focusing distance and provides more ergonomic position for the providers and patients but is currently available at limited practices only. A high definition Olympus ' X190 series colonoscope has a 170 -viewing angle with 5 to $100 \mathrm{~mm}$ depth of field. Depending on the screen size it provides up to 80 times magnification. The anal area can be evaluated effectively same as the operating microscope by using a colonoscope (Figure 5). The biopsy can be performed using "through the scope" biopsy forceps. The examination at first is limited due to inability to use the acetic acid and the Lugol's iodine stain while reviewing the anal area. The colonoscope is withdrawn and if needed the acetic acid and the Lugol's iodine is reapplied. In the initial process of defining the protocol for the eHRA, the authors had foreseen difficulty in identifying the anatomical location of the lesion; hence the anoscope opposed to the right anal canal was marked with the surgical marker. The procedures were only performed in conjunction with the colonoscopy. In view of endoscope used in our technique, this protocol is limited to a gastroenterologist performing the HRA.

Even though there are several similarities between cervical and anal examination, there are prominent differences making HRA challenging. The difficult anal anatomy in form of the hypertrophied anal papillae and hemorrhoids pose technical difficulties in performing the HRA [22]. Moreover, identifying high-grade lesions in the anal canal is hampered due to the tight skin folds obscuring the lower anal canal. Usually the anal skin at the ano-rectal transition zone is opposing the transparent plastic bevel of the anoscope. An endoscope,

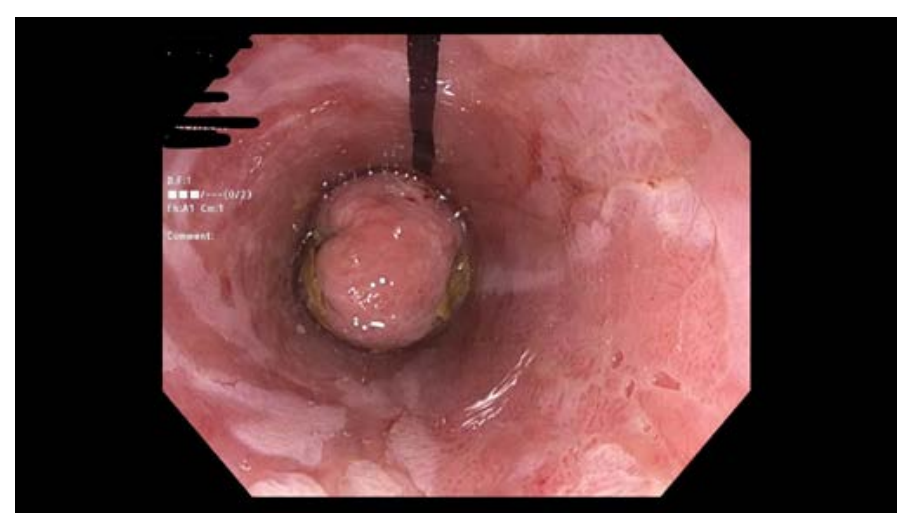

Figure 5. Demonstrates circumferential view of the ano-rectal transistion zone through the anoscope

with varying focus distance, can view the entire squamo-columnar epithelium through the transparent anoscope and the lesion can be well appreciated in the skin fold (Figure 6). We define this as the circumferential view, it provides a better evaluation in the patient with the altered anal anatomy. The conventional HRA does not provide this view. The authors cannot provide an opinion about the experience with video ProctoStation (Balmer Medical ${ }^{\circ}$ ) due to its unavailability at our center.

The use of anoscope can involuntarily increase the anal tone, and thus patient discomfort which may hinder the evaluation. The pain and discomfort during the procedure is generally well tolerated. However, selected cases may need the monitored conscious sedation [30]. In view of eHRA performed in conjunction with colonoscopy, our procedures were performed under the monitored anesthesia care, which may aid in evaluation that is more thorough to increase our yield.

The rates of the HPV related anal cancer in HIV positive population have increased (70 to 100 per 100,000) [31]. These rates are comparable to the colon cancer prevalence. With the rigorous screening protocol, the rate of the colon cancer has decreased over time [32]. These changes can be attributed to the early diagnosis and management of the high-grade adenoma of the colon [33]. In the field of anal cancer, "The ANCHOR Study" aims at uncovering the benefits of treating the pre-cancerous lesion- HSIL. If the ANCHOR study results are positive and they indeed show reduction in anal cancer rates by implementing early management of HSIL, there will be a deficiency of experienced anoscopists who can perform HRA [34]. Hence, utilizing an endoscope to assist in HRA can bring in several other gastroenterologists on board to fight against rising rates of anal cancer.

\section{Conclusion}

In summary, the endoscope assisted high resolution anoscopy (eHRA) improves detection of anal dysplasia in high risk patients with HSIL on anal cytology. Utilizing an endoscope overcomes the limitation of inexperience with a viewing microscope and helps the gastroenterologist perform a comprehensive anal examination.

\section{Author contributions}

Harish Patel, Hassan Tariq and Jasbir Makker contributed to concept and design. Harish Patel drafted manuscript. Rafeeq Ahmed and Chaitanya Chandrala contributed to acquisition and analysis of the data. Mariela Glandt, Jabir Makker and Sridhar Chilimuri contributed to critical revision of the manuscript for important intellectual content. 


\section{Institutional review board statement}

The study was performed in agreement with the ethical guidelines of the Declaration of Helsinki and the protocol was approved by the local Ethics Committee (IRN\# 0214 19 04)

\section{Conflict-of-interest statement}

The authors declare that they have no conflict of interest.

\section{References}

1. Lieberman DA (2012) Guidelines for colonoscopy surveillance after screening and polypectomy: a consensus update by the US Multi-Society Task Force on Colorectal Cancer. Gastroenterology 143: 844-857. [Crossref]

2. Albuquerque A (2015) High-resolution anoscopy: Unchartered territory for gastroenterologists? World J Gastrointest Endosc 7: 1083-1087. [Crossref]

3. Masur H (2014) Prevention and treatment of opportunistic infections in HIV-infected adults and adolescents: Updated Guidelines from the Centers for Disease Control and Prevention, National Institutes of Health, and HIV Medicine Association of the Infectious Diseases Society of America. Clin Infect Dis 58: 1308-1311. [Crossref]

4. Chin-Hong PV, Palefsky JM (2002) Natural history and clinical management of anal human papillomavirus disease in men and women infected with human immunodeficiency virus. Clin Infect Dis 35: 1127-1134. [Crossref]

5. Swedish KA, EQ Lee, SE Goldstone (2011) The changing picture of high-grade anal intraepithelial neoplasia in men who have sex with men: the effects of 10 years of experience performing high-resolution anoscopy. Dis Colon Rectum 54: 1003-1007. [Crossref]

6. Fennerty MB (1994) Tissue staining. Gastrointest Endosc Clin N Am 4: 297-311.

7. Leonard D, Beddy D, Dozois EJ (2011) Neoplasms of anal canal and perianal skin. Clin Colon Rectal Surg 24: 54-63. [Crossref]

8. Lenselink CH (2008) Sexual behaviour and HPV infections in 18 to 29-year-old women in the pre-vaccine era in the Netherlands. PLoS One 3: e3743. [Crossref]

9. Nelson RA (2013) Changing patterns of anal canal carcinoma in the United States. $J$ Clin Oncol 31: 1569-1575. [Crossref]

10. Myerson RJ, Karnell LH, Menck HR (1997) The National Cancer Data Base report on carcinoma of the anus. Cancer 80: 805-815. [Crossref]

11. Hernandez-Ramirez RU (2017) Cancer risk in HIV-infected people in the USA from 1996 to 2012: a population-based, registry-linkage study. Lancet HIV 4: e495-e504. [Crossref]

12. Yang EJ (2015) Microanatomy of the cervical and anorectal squamocolumnar junctions: a proposed model for anatomical differences in HPV-related cancer risk. Mod Pathol 28: 994-1000. [Crossref]

13. Pineda CE, Welton ML (2008) Controversies in the management of anal high-grade squamous intraepithelial lesions. Minerva Chir 63: 389-399. [Crossref]

14. Panther LA, Schlecht HP, Dezube BJ (2005) Spectrum of human papillomavirusrelated dysplasia and carcinoma of the anus in HIV-infected patients. AIDS Read 15: $79-91$.
15. Crum-Cianflone NF (2010) Anal cancers among HIV-infected persons: HAART is not slowing rising incidence. AIDS 24: 535-543. [Crossref]

16. Noone AM, Krapcho HN, Miller D, Brest A, Yu M, et al. (2014) SEER Cancer Statistics Review, 1975-2015, National Cancer Institute. Bethesda, April 2018.

17. Massad LS (2013) 2012 Updated consensus guidelines for the management of abnormal cervical cancer screening tests and cancer precursors. Obstet Gynecol 121: 829-846. [Crossref]

18. Chiao EY (2006) Screening HIV-infected individuals for anal cancer precursor lesions: a systematic review. Clin Infect Dis 43: 223-233. [Crossref]

19. Steele SR (2012) Practice parameters for anal squamous neoplasms. Dis Colon Rectum 55: 735-749. [Crossref]

20. Palefsky JM (1997) Anal cytology as a screening tool for anal squamous intraepithelia lesions. J Acquir Immune Defic Syndr Hum Retrovirol 14: 415-422. [Crossref]

21. Cambou MC (2015) Anal human papillomavirus (HPV) prevalences and factors associated with abnormal anal cytology in HIV-infected women in an urban cohort from Rio de Janeiro, Brazil. AIDS Patient Care STDS 29: 4-12. [Crossref]

22. Palefsky JM (2012) Practising high-resolution anoscopy. Sex Health 9: 580-586 [Crossref]

23. Darragh TM (2012) The Lower Anogenital Squamous Terminology Standardization Project for HPV-Associated Lesions: background and consensus recommendations from the College of American Pathologists and the American Society for Colposcopy and Cervical Pathology. Arch Pathol Lab Med 136: 1266-1297. [Crossref]

24. Malebranche R (1983) Acquired immunodeficiency syndrome with severe gastrointestinal manifestations in Haiti. Lancet 2: 873-878.

25. Dworkin B (1985) Gastrointestinal manifestations of the acquired immunodeficiency syndrome: a review of 22 cases. Am J Gastroenterol 80: 774-778. [Crossref]

26. Corley DA, Cello JP, Koch J (1999) Evaluation of upper gastrointestinal tract symptoms in patients infected with HIV. Am J Gastroenterol 94: 2890-2896. [Crossref]

27. Crum-Cianflone NF (2010) HIV and the Gastrointestinal Tract. Infect Dis Clin Pract (Baltim Md) 18: 283-285. [Crossref]

28. Palefsky JM (2009) Anal cancer prevention in HIV-positive men and women. Curr Opin Oncol 21: 433-438.

29. Hillman RJ (2016) 2016 IANS International Guidelines for Practice Standards in the Detection of Anal Cancer Precursors. J Low Genit Tract Dis 20: 283-291. [Crossref]

30. De-Masi A (2018) The acceptability of high resolution anoscopy examination in patients attending a tertiary referral centre. BMC Cancer 18: 554. [Crossref]

31. Dandapani SV (2010) HIV- positive anal cancer: an update for the clinician. $J$ Gastrointest Oncol 1: 34-44. [Crossref]

32. Group USCSW (2014) U.S. Cancer Statistics Data Visualizations Tool, based on November 2017 submission data (1999-2015). U.S. Department of Health and Human Services, Centers for Disease Control and Prevention and National Cancer Institute, June 2018.

33. Zauber AG (2012) Colonoscopic Polypectomy and Long-Term Prevention of Colorectal-Cancer Deaths. N Engl J Med 366: 687-696. [Crossref]

34. Richel O, Prins JM, de Vries HJ (2014) Screening for anal cancer precursors: what is the learning curve for high-resolution anoscopy? AIDS 28: 1376-1377. [Crossref]

Copyright: (C2019 Patel H. This is an open-access article distributed under the terms of the Creative Commons Attribution License, which permits unrestricted use, distribution, and reproduction in any medium, provided the original author and source are credited. 